\title{
On the Structure of Analytic Renormalization
}

\author{
EUGENE R. SPEER \\ Department of Mathematics, Massachusetts Institute of Technology, Cambridge, Mass.
}

Received April 2, 1971

Abstract. A direct proof is given that analytic renormalization has an additive structure and hence may be implemented by counterterms in the Lagrangian.

\section{§ 1. Introduction}

Of the various types of renormalization in perturbative quantum field theory we will here accept as basic the additive approach of Bogoliubov [1]. In this method formally infinite quantities are subtracted from a divergent Feynman amplitude to produce a finite renormalized result. Physics enters because these subtractions may be implemented in the field theory by inserting counterterms into the interaction Lagrangian; the counterterms are then related to renormalizations of mass, charge, etc.

The particular implementation of the additive aproach given by Bogoliubov has two difficulties: the recursive definition of the renormalized amplitudes makes them difficult to compute, and the proof that the result is finite is very complicated (see [1-3]). Analytic renormalization $([4,5])$ removes these difficulties, to a large extent, by giving a simple prescription which obviously yields a finite result. On the other hand, to establish the connection with physics (specifically, to show that analytic renormalization is equivalent to additive renormalization) it was necessary in $[4,5]$ to use the recursive definition as a starting point. In the present paper we avoid this problem and show directly that analytic renormalization may be implemented by counterterms in the Lagrangian.

Some work related to these goals has previously appeared. In [6] Westwater gives a definition of analytic renormalization which he shows to be implementable by counterterms (and to be equivalent to the definition of [4, 5]). His definition is not recursive, but, like the Bogoliubov prescription, does involve modifying the integral by modification of (i.e., subtractions from) the integrand. The present paper is more in the spirit of $[4,5]$; the counterterms, like the renormalized amplitude itself, 
are obtained from well defined operators on a space of meromorphic functions. In [7], Hepp gives an axiomatic formulation of renormalization and shows easily that analytic renormalization satisfies the axioms. However, his proof that the axioms may also be implemented by an additive procedure rests on the usual recursively defined counterterms. It is hoped that the present paper may provide an easier ascent of this "Aiquille Verte“.

In $\S 2$ we review briefly the basic results of the theory of analytic renormalization (following the notation of [5]) and the formulation of additive renormalization which will be convenient here. In $\S 3$ we study a natural decomposition of meromorphic functions in a certain class, and in $\S 4$ show that on the functions of physical interest this corresponds to the counterterms of additive renormalization. We do not discuss the question of finite renormalization, which is thoroughly dealt with in $[7,8]$.

\section{§ 2. Review}

We recall the following terminology. A Feynman graph $G$ is a graph (with vertices $V_{1}, \ldots V_{m}$ and set of lines $\mathscr{L}=\mathscr{L}(G)$ ), to each line $\ell$ of which there is associated a propagator $\Delta_{\ell} \in \mathscr{S}^{\prime}\left(\mathbb{R}^{4}\right)$ whose Fourier transform has the form

$$
\tilde{\Delta}_{\ell}(p)=Z_{\ell}(p)\left(p^{2}-m_{\ell}^{2}+i 0\right)^{-1} .
$$

Here $Z_{\ell}$ is a polynomial of degree $r_{\ell}$, and $m_{\ell}>0$ is the mass associated with the line. For our purposes all vertices of $G$ are considered to be external. The superficial divergence of $G$ is

$$
\mu(G)=\sum_{\ell \in \mathscr{L}}\left(r_{\ell}+2\right)-4(m-1) .
$$

A subgraph $H \subset G$ is again a Feynman graph in an obvious way.

The Feynman graph $G$ is irreducible if it is connected and cannot be disconnected by removing a single line or vertex. A singularity family (s-family) $\mathscr{E}$ for $G$ is a collection of irreducible subgraphs of $G$ such that (a) if $H, H^{\prime} \in \mathscr{E}$, then $H \supset H^{\prime}, H^{\prime} \supset H$, or $\mathscr{L}(H) \cap \mathscr{L}\left(H^{\prime}\right)=\emptyset$, and (b) if $H_{1}, \ldots H_{k} \in \mathscr{E}$ satisfy $\mathscr{L}\left(H_{i}\right) \cap \mathscr{L}\left(H_{j}\right)=\emptyset$ for any $i, j$, then $H=H_{1} \cup \ldots \cup H_{k}$ is not irreducible. (We note that this terminology differs slightly from that of [5], in which an s-family was required to be a maximal family satisfying (a) and (b).)

Now let $G_{0}$ be a connected Feynman graph with $n$ vertices. For each line $\ell$ of $G_{0}$ we introduce a complex parameter $\lambda_{\ell}$ and a new pro- 
pagator

$$
\tilde{\Delta}_{\ell}\left(\lambda_{\ell}\right)(p)=Z_{\ell}(p)\left(p^{2}-m^{2}+i 0\right)^{-\lambda_{\ell}} .
$$

The generalized Feynman amplitude for $G_{0}$ is the distribution $\mathscr{T}_{G_{0}}(\lambda) \in \mathscr{S}^{\prime}\left(\mathbb{R}^{4 n}\right)$, depending meromorphicly on $\lambda$, which for sufficiently large $\operatorname{Re} \lambda_{\ell}$ is given by

$$
\mathscr{T}_{G_{0}}(\lambda)=\prod_{\ell \in \mathscr{L}\left(\mathbf{G}_{0}\right)} \Delta_{\ell}\left(\lambda_{\ell}\right)\left(x_{f_{\ell}}-x_{i_{\ell}}\right),
$$

with $V_{i \ell}, V_{f_{\ell}}$ the initial and final vertices of $\ell$ in $G_{0}$. Thus $\mathscr{T}_{G_{0}}(\lambda)$ is formally equal to the usual Feynman amplitude for $\lambda=\lambda_{0}=(1,1, \ldots 1)$. The analytic structure of $\mathscr{T}_{G_{0}}(\lambda)$ may be described as follows: there is a (nonunique) decomposition

$$
\mathscr{T}_{G_{0}}(\lambda)=\sum_{\mathscr{E}} \mathscr{T}_{G_{0}}(\lambda, \mathscr{E}),
$$

the sum taken over all maximal $s$-families for $G_{0}$, such that $\mathscr{T}_{G_{0}}(\lambda, \mathscr{E})$ is regular except for simple poles on the varieties

$$
\sum_{\ell \in \mathscr{L}(G)}\left(\lambda_{\ell}-1\right)=\left[\frac{\mu(G)}{2}\right]-k,
$$

with $G \in \mathscr{E}$ and $k$ a non-negative integer.

In particular, if the superficial divergence of any subgraph is positive, $\mathscr{T}_{G_{0}}$ with be singular at $\lambda_{0}$. The extraction of an appropriate finite part at this singular point is called analytic renormalization. This is done using a generalized evaluator:

Definition 2.3. Let $j(\lambda)=\prod_{\mathscr{K}}\left[\sum_{\ell \in \mathscr{K}}\left(\lambda_{\ell}-1\right)\right]$, the product taken over all non-empty $\mathscr{K} \in \mathscr{L}$. For $\varepsilon>0$, define $U_{\varepsilon}=\left\{\lambda|| \lambda_{\ell}-1 \mid<\varepsilon, \ell \in \mathscr{L}\right\}$, and $\mathscr{A}_{\varepsilon}=\left\{f(\lambda) \mid f(\lambda) j(\lambda)\right.$ is analytic in $\left.U_{\varepsilon}\right\} . \mathscr{A}_{\varepsilon}$ is topologized by uniform convergence, on compact subsets of $U_{\varepsilon}$, of the products $j(\lambda) f(\lambda), f \in \mathscr{A}_{\varepsilon}$. Let $\mathscr{A}=\bigcup_{\varepsilon>0} \mathscr{A}_{\varepsilon}$. Then a map $\mathscr{W}: \mathscr{A} \rightarrow \mathbb{C}$ is a generalized evaluator if the following conditions are satisfied:

(W 1) $\mathscr{W}$ is linear;

(W 2) if $f \in \mathscr{A}$ is analytic at $\lambda_{0}$, then $\mathscr{W} f=f\left(\lambda_{0}\right)$;

(W 3 ) for any $\varepsilon>0, \mathscr{W}$ is continuous on $\mathscr{A}_{\varepsilon}$;

(W 4) if $s$ is any permutation of $\mathscr{L}$, and if for $f \in \mathscr{A}, f_{s} \in \mathscr{A}$ is defined by $f_{s}\left(\lambda_{\ell_{1}}, \ldots\right)=f\left(\lambda_{s\left(\ell_{1}\right)}, \ldots\right)$, then $\mathscr{W} f_{s}=\mathscr{W} f$

(W 5) if $f_{1}, f_{2} \in \mathscr{A}$ depend only on $\left\{\lambda_{\ell} \mid \ell \in \mathscr{L}_{1}\right\}$ and $\left\{\lambda_{\ell} \mid \ell \in \mathscr{L}_{2}\right\}$, respectively, with $\mathscr{L}_{1} \cap \mathscr{L}_{2}=\emptyset$, then $\mathscr{W}\left(f_{1} f_{2}\right)=\mathscr{W} f_{1} \mathscr{W} f_{2}$.

Now if $T(\lambda)$ is a distribution such that $T(\lambda)(\psi)$ is in $\mathscr{A}$, for any test function $\psi$, we may define a distribution $\mathscr{W} T$ by $\mathscr{W} T(\psi)=\mathscr{W}(T(\psi))$. This justifies 
Definition 2.4. For any generalized evaluator $\mathscr{W}$, the distribution $\mathscr{W}_{G_{0}} \in \mathscr{S}^{\prime}\left(\mathbb{R}^{4 n}\right)$ is called an analytically renormalized Feynman amplitude for $G_{0}$.

For a discussion of additive renormalization we must recall some additional terminology. A generalized vertex is a non-empty subset $\left\{V_{1}^{\prime}, \ldots V_{m}^{\prime}\right\}$ of the vertices of $G_{0}$; the graph $G\left(V_{1}^{\prime}, \ldots V_{m}^{\prime}\right)$ is the subgraph of $G_{0}$ consisting of all lines joining any pair of vertices in the subset. A vertex part for $\left\{V_{1}^{\prime}, \ldots V_{m}^{\prime}\right\}$ is a distribution $\mathscr{X}\left(\lambda ; V_{1}^{\prime}, \ldots V_{m}^{\prime}\right)$, depending only on $\left\{\lambda_{\ell} \mid \ell \in \mathscr{L}\left(G\left(V_{1}^{\prime}, \ldots V_{m}^{\prime}\right)\right)\right\}$, and having the form

$$
\tilde{\mathscr{X}}\left(\lambda ; V_{1}^{\prime}, \ldots V_{m}^{\prime}\right)=\left\{\begin{array}{l}
1, \text { if } m=1 ; \\
0, \text { if } G\left(V_{1}^{\prime}, \ldots V_{m}^{\prime}\right) \text { is not IPI } \\
\delta\left(\sum_{1}^{m} p_{i}^{\prime}\right) P\left(\lambda ; p_{1}^{\prime}, \ldots p_{m}^{\prime}\right) \text { otherwise. }
\end{array}\right.
$$

Here $P\left(\lambda ; p_{1}^{\prime}, \ldots p_{m}^{\prime}\right)$ is a polynomial in the $p_{i}^{\prime}$ of degree at most $\mu\left(G\left(V_{1}^{\prime}, \ldots V_{m}^{\prime}\right)\right)$; we will assume that for fixed $p_{i}^{\prime}, P \in \mathscr{A}$. An IPI graph is one which is connected and cannot be disconnected by the removal of one line.

Now suppose that we have assigned a vertex part $\mathscr{X}$ to each generalized vertex. Let $P$ be a partition of $\left\{V_{1}, \ldots V_{n}\right\}$ into $k$ generalized vertices $\left\{V_{i, 1}^{P}, \ldots V_{i, m(i)}^{P}\right\}, i=1, \ldots k$. We may define the amplitude $\mathscr{T}_{P, x}(\lambda)$ to be given, for $\operatorname{Re} \lambda_{\ell}$ sufficiently large, by

$$
\mathscr{T}_{P, \mathscr{X}}(\lambda)=\prod_{\ell \in \mathscr{L}^{\prime}} \Delta_{\ell}\left(\lambda_{\ell}\right)\left(x_{f_{\ell}}-x_{i_{\ell}}\right) \prod_{i=1}^{k} \mathscr{X}\left(\lambda ; V_{i, 1}^{P}, \ldots\right) ;
$$

and for other values of $\lambda$ by analytic continuation.

$$
\left(\text { Here } \mathscr{L}^{\prime}=\mathscr{L}-\bigcup_{i=1}^{k} \mathscr{L}\left(G\left(V_{i, 1}^{P}, \ldots\right)\right) .\right) \text {. }
$$

Definition 2.5. A distribution $T \in \mathscr{S}^{\prime}\left(\mathbb{R}^{4 n}\right)$ is an additively renormalized Feynman amplitude for $G_{0}$ if there exists a set of vertex parts $\mathscr{X}(\lambda)$ such that

$$
T=\lim _{\lambda \rightarrow \lambda_{0}} \sum_{P} \mathscr{T}_{P, x}(\lambda)
$$

the sum taken over all partitions of $\left\{V_{1}, \ldots V_{n}\right\}$.

In Section 4 we will show that every analytically renormalized amplitude for $G_{0}$ is an additively renormalized amplitude. We note that, once the existence of the vertex part $\mathscr{X}$ has been established, we may in the additive formalism set all $\lambda_{\ell}$ equal to some complex variable $\lambda$; this is more convenient for the introduction of counterterms in the Lagrangian (see [6]). 


\section{§ 3. Analytic Decomposition}

Take $M>0$, and let $\Omega$ denote the index set $\{1, \ldots M\}$. A family $\mathscr{E}$ of non-empty subsets of $\Omega$ is called an s-family if (a) $\chi_{1}, \chi_{2} \in \mathscr{E}$ implies that either $\chi_{1} \subset \chi_{2}, \chi_{2} \subset \chi_{1}$, or $\chi_{1} \cap \chi_{2}=\emptyset$, and (b) for any $\chi \in \mathscr{E}$,

$$
\chi \neq \bigcup_{\chi^{\prime} \in \mathscr{E}, \chi_{\ddagger}^{\prime} \subsetneq \chi} \chi^{\prime} .
$$

Let $\mathscr{E}$ be an $s$-family. We let $\overline{\mathscr{E}}=\bigcup_{\chi \in \mathscr{E}} \chi \cdot \mathscr{E}$ is discrete if all elements of $\mathscr{E}$ are pairwise disjoint. For any $\mathscr{F} \subset \mathscr{E}, \mathscr{E}(\mathscr{F})$ is the subfamily of $\mathscr{E}$ consisting of all sets which are proper subsets of some element of $\mathscr{F}$; for $\chi \in \mathscr{E}$ we write $\mathscr{E}(\chi)$, etc., instead of $\mathscr{E}(\{\chi\})$.

We will consider functions defined on $\mathbb{C}^{\Omega}\left(=\mathbb{C}^{M}\right)$. For $\chi \subset \Omega$ there is a natural decomposition $\mathbb{C}^{\Omega}=\mathbb{C}^{\chi} \oplus \mathbb{C}^{\Omega-\chi}$, and we write correspondingly $\lambda=\lambda^{\chi} \oplus \lambda^{\Omega-\chi}$ for any $\lambda \in \mathbb{C}^{\Omega}$. As usual, $\lambda_{0} \in \mathbb{C}^{\Omega}$ is the point $(1, \ldots 1)$. For $\chi \subset \Omega$ we write $\Lambda(\chi)=\sum_{i \in \chi}\left(\lambda_{i}-1\right)$; for any s-family $\mathscr{E}, \Lambda(\mathscr{E})=\prod_{\chi \in \mathscr{E}} \Lambda(\chi)$.

We now introduce a new class of operators closely related to generalized evaluators.

Definition 3.2. Let $\mathscr{A}, \mathscr{A}_{\varepsilon}$ be as in Def. 2.3 (with $\mathscr{L}$ replaced by $\Omega$ ). $\mathscr{B} \subset \mathscr{A}$ is the subspace of all functions $f$ having a (not necessarily unique) decomposition

$$
f(\lambda)=\sum_{\mathscr{E}} f(\lambda, \mathscr{E}),
$$

the sum taken over all s-families $\mathscr{E}$, with $\Lambda(\mathscr{E}) f(\lambda, \mathscr{E})$ analytic at $\lambda_{0}$. $\mathscr{B}_{0} \subset \mathscr{B}$ consists of all $f$ which are analytic at $\lambda_{0}$. An analytic evaluator is a map $\mathscr{V}: \mathscr{B} \rightarrow \mathscr{B}_{0}$ such that:

(V 1) $\mathscr{V}$ is linear;

(V 2) if $f$ is in $\mathscr{B}_{0}, \mathscr{V} f=f$;

(V 3) for any $\varepsilon>0, \mathscr{V}$ is continuous on $\mathscr{B} \cap \mathscr{A}_{\varepsilon}$;

(V 4) if $s$ is any permutation on $\Omega, \mathscr{V} f_{s}=(\mathscr{V} f)_{s}$;

(V 5) if $f_{1}, f_{2} \in \mathscr{B}$ depend on disjoint sets of $\lambda$ 's, then $\mathscr{V}\left(f_{1} f_{2}\right)=\mathscr{V} f_{1} \mathscr{V} f_{2}$; (V 6) if $f \in \mathscr{B}$ is independent of $\lambda_{i}$, so is $\mathscr{V} f$.

Remark 3.4. (a) If we compare Definitions 2.3 and 3.2 we see that the significant difference between analytic and generalized evaluators lies in conditions (V 2) and (W 2). If $\mathscr{V}$ is any analytic evaluator, the operator $\mathscr{W}: \mathscr{B} \rightarrow \mathbb{C}$ defined by $\mathscr{W} f=\mathscr{V} f\left(\lambda_{0}\right)$ satisfies (W 1$)-(\mathrm{W} 5)$ and is thus, aside from its smaller domain of definition, a generalized evaluator. We will prove a converse of this statement in the Appendix.

(b) An example of an analytic evaluator is easily given. For $f \in \mathscr{B} \cap \mathscr{A}_{\varepsilon}$, choose $0<R_{1}<\cdots<R_{n}<\varepsilon$ to satisfy $R_{i}>\sum_{j<i} R_{j}$, and let $C_{i}$ be the 
contour $|z-1|=R_{i}$ oriented counterclockwise. Then for $\left|\lambda_{i}-1\right|<R_{1}$ define

$$
\mathscr{V}_{0} f(\lambda)=\frac{(2 \pi i)^{-M}}{M !} \sum_{s} \int_{C_{s(1)}} d \mu_{1} \ldots \int_{C_{s(M)}} d \mu_{M} \frac{f(\boldsymbol{\mu})}{\left(\mu_{1}-\lambda_{1}\right) \ldots\left(\mu_{M}-\lambda_{M}\right)},
$$

the sum running over all permutations $s$ of $\Omega$.

An analytic evaluator $\mathscr{V}$ may be used to remove the singularity of a function which is associated with the $\lambda$ variables in a subset $\chi \subset \Omega$, by treating the other variables like constants when we apply $\mathscr{V}$. This is formalized in

Definition 3.5. For $\boldsymbol{\mu} \in \mathbb{C}^{\Omega-\chi}$ and $f \in \mathscr{B}$ define $f_{\mu} \in \mathscr{B}$ by $f_{\mu}(\lambda)=f\left(\lambda^{\chi} \oplus \boldsymbol{\mu}\right)$. Then $\mathscr{V}_{\chi}: \mathscr{B} \rightarrow \mathscr{B}$ is defined by

$$
\mathscr{V}_{\chi} f(\lambda)=\left(\mathscr{V} f_{\lambda^{s-\chi}}\right)(\lambda) .
$$

$\mathscr{V}_{\chi} f$ is regular at (generic) points of $\left\{\lambda \mid \lambda_{i}=1, i \in \chi\right\} ; \mathscr{V}_{\chi}$ satisfies (V 1)-(V 6) with slight modifications: in (V 4) we must assume $s(\chi)=\chi$, while in (V 2) we need only assume $f$ regular on $\left\{\lambda \mid \lambda_{i}=1, i \in \chi\right\}$. Note that $\mathscr{V}_{\Omega}=\mathscr{V}$, and that $\mathscr{V}_{\emptyset}$ is the identity.

In the remainder of this section we deal with some fixed analytic evaluator $\mathscr{V}$.

Lemma 3.6. Suppose $\chi \subset \Omega$, and $\psi_{1}, \ldots \psi_{k} \subset \chi$ are pairwise disjoint subsets of $\chi$. Suppose $f \in \mathscr{B}$ is such that $f$ is not singular on any $\left\{\Lambda\left(\chi^{\prime}\right)=0\right\}$ with $\chi^{\prime} \subset \chi$ unless $\chi^{\prime} \subset \psi_{i}$, for some $i$. Then

$$
\mathscr{V}_{\chi} f=\left[\prod_{i=1}^{k} \mathscr{V}_{\psi_{i}}\right] f
$$

and the operators $\mathscr{V}_{\psi_{i}}$ commute on $f$.

Proof. The proof is representative of methods used to deal with analytic evaluators. Consider the function

$$
g(\lambda)=\prod_{i=1}^{k} \prod_{\emptyset \neq \psi_{i}^{\prime} \subset \psi_{i}} \Lambda\left(\psi_{i}^{\prime}\right) f(\lambda) .
$$

If $\boldsymbol{\mu} \in \mathbb{C}^{M}$ is a generic point with $\mu_{i}=1$ for all $i \in \chi, g$ is regular at $\mu$. By expanding $g$ in a Taylor series at $\boldsymbol{\mu}$ we may write $g$, and hence $f$, as the sum of a series, each term of which is the product of factors depending on $\lambda^{\psi_{i}}, \lambda^{\chi-\cup \psi_{i}}$, and $\lambda^{\Omega-\chi}$. If $f$ is actually such a product, (3.7) follows immediately from (V 5) and (V 6); for general $f$ it follows from the series expansion and the continuity property (V 3$)$.

Lemma 3.8. Let $\mathscr{E}$ be a fixed s-family. Then for any $f \in \mathscr{B}$, with $\Lambda(\mathscr{E}) f$ analytic at $\lambda_{0}$,

$$
\mathscr{V} f=\sum_{\mathscr{D}} \prod_{\chi \in \mathscr{D}}\left(\mathscr{V}_{\chi}-\mathscr{V}_{\bar{\delta}(\chi)}\right) f
$$

the sum running over all discrete subfamilies $\mathscr{D} \subset \mathscr{E}$. 
Proof. The proof is by straight forward rearrangement of terms in (3.9). For any $\mathscr{D} \subset \mathscr{E}$, we let $\mathscr{E}^{0}(\mathscr{D}) \subset \mathscr{E}$ consist of all maximal elements of $\mathscr{E}(\mathscr{D})$. If we expand the product over $\chi$, the right hand side of (3.9) becomes

$$
\sum_{\mathscr{D}} \sum_{\mathscr{F} \subset \mathscr{D}}(-1)^{|\mathscr{F}|} \mathscr{V}_{\overline{\mathscr{D}}} f
$$

where we have set

$$
\mathscr{D}^{\prime}=\mathscr{E}^{0}(\mathscr{F}) \cup(\mathscr{D}-\mathscr{F})
$$

and used Lemma 3.6. $\mathscr{D}^{\prime}$ is a discrete subset of $\mathscr{E}$.

Now for any fixed (discrete) $\mathscr{D}^{\prime} \subset \mathscr{E}$, let $\mathscr{D}_{1}^{\prime}=\left\{\chi \in \mathscr{E} \mid \mathscr{E}^{0}(\chi) \subset \mathscr{D}^{\prime}\right.$, $\left.\mathscr{E}^{0}(\chi) \neq \emptyset\right\}$, and $\mathscr{D}_{2}^{\prime}=\left\{\chi \in \mathscr{E} \mid \mathscr{E}^{0}(\chi)=\emptyset, \chi \notin \mathscr{D}^{\prime} \cup \mathscr{E}\left(\mathscr{D}^{\prime}\right)\right\}$. Then there is a biunique correspondence between pairs $(\mathscr{D}, \mathscr{F})$ occurring in (3.10) and pairs $\left(\mathscr{D}^{\prime}, \mathscr{F}\right)$ with $\mathscr{F} \subset \mathscr{D}_{1}^{\prime} \cup \mathscr{D}_{2}^{\prime}$, given by (3.11) and, in the opposite direction, by

$$
\mathscr{D}=\mathscr{F} \cup\left(\mathscr{D}^{\prime}-\mathscr{E}^{0}(\mathscr{F})\right) .
$$

Thus (3.10) may be rewritten

$$
\sum_{\mathscr{D}^{\prime}} \mathscr{V}_{\overline{\mathscr{D}}^{\prime}}\left[\sum_{\mathscr{F}}(-)^{|\mathscr{F}|}\right] f=\sum_{\mathscr{D}^{\prime}}^{\prime} \mathscr{V}_{\overline{\mathscr{D}}} f
$$

where $\Sigma^{\prime}$ runs over those discrete $\mathscr{D}^{\prime} \subset \mathscr{E}$ such that $\mathscr{D}_{1}^{\prime}=\mathscr{D}_{2}^{\prime}=\emptyset$. But the only such $\mathscr{D}^{\prime}$ consists precisely of all maximal elements of $\mathscr{E}$. (3.9) now follows from Lemma 3.6.

Corollary 3.12. For $f \in \mathscr{B}$, let $f=\sum_{\mathscr{E}} f(\cdot, \mathscr{E})$ be a decomposition as in
3). Then (3.3). Then

$$
\mathscr{V} f=\sum_{\chi \subset \Omega} \sum_{(\mathscr{E}, \mathscr{D})} \prod_{\psi \in \mathscr{D}}\left(\mathscr{V}_{\psi}-\mathscr{V}_{\overline{\mathscr{E}}(\psi)}\right) f(\cdot, \mathscr{E}),
$$

where the second sum is over all s-families $\mathscr{E}$ such that for some discrete $\mathscr{D} \subset \mathscr{E}, \overline{\mathscr{D}}=\chi$.

Proof. Immediate.

This result motivates the basic

Definition 3.14. For any $\chi \subset \Omega$, we define $\mathscr{S}(\chi): \mathscr{B} \rightarrow \mathscr{B}$ by

$$
\mathscr{S}(\chi) f=\sum_{(\mathscr{E}, \mathscr{D})} \prod_{\psi \in \mathscr{D}}\left(\mathscr{V}_{\psi}-\mathscr{V}_{\overline{\mathscr{E}}(\psi)}\right) f(\cdot, \mathscr{E}) ;
$$

where $f=\Sigma f(\cdot, \mathscr{E})$ as in (3.3), and again the sum is over all $\mathscr{E}$ such that, for some discrete $\mathscr{D} \subset \mathscr{E}, \overline{\mathscr{D}}=\chi . \mathscr{S}(\chi) f$ is called the singular part of $f$ associated with $\chi$. We note that $\mathscr{S}(\emptyset)$ is the identity (since, if $\chi=\emptyset$, all $s$-families $\mathscr{E}$ appear in (3.15), $\mathscr{D}=\emptyset$ in each case, and the empty product is by convention 1$)$. 
Lemma 3.16. $\mathscr{S}(\chi)$ is well defined.

Proof. By Lemma 3.6, no ambiguity arises from our failure to specify the order of $\mathscr{V}$ factors in the product in (3.15). There remains the problem that the decomposition (3.3) of $f$ is not unique. Suppose then that we have two such decompositions

$$
f(\lambda)=\Sigma f_{1}(\lambda, \mathscr{E})=\Sigma f_{2}(\lambda, \mathscr{E}) .
$$

We denote the corresponding values of (3.15) by $\mathscr{S}_{1}(\chi) f$ and $\mathscr{S}_{2}(\chi) f$, respectively, and wish to show that

$$
\mathscr{S}_{1}(\chi) f=\mathscr{S}_{2}(\chi) f .
$$

Let $\zeta \subset \Omega$ be the smallest set such that $f, f_{1}(\cdot, \mathscr{E})$, and $f_{2}(\cdot, \mathscr{E})$ depend only on the variables $\lambda^{\zeta}$. We prove (3.18) by induction on $|\zeta|$; the case $|\zeta|=M$ is the desired conclusion. (Note that even if $|\zeta|<M$, the sums in (3.17) may still run over all $s$-families.) As a preliminary remark we observe that $\mathscr{S}_{i}(\chi) f=0$ unless $\chi \subset \zeta(i=1,2)$. This is because, if $\chi-\zeta \neq \emptyset$, then for any $(\mathscr{E}, \mathscr{D})$ in $(3.15)$ there will be a $\psi \in \mathscr{D}$ with $\psi-\zeta \neq \emptyset$, and then, by Lemma 3.6, $\left(\mathscr{V}_{\psi}-\mathscr{V}_{\overline{\mathscr{E}(\psi)}}\right) f_{i}(\cdot, \mathscr{E})=0$. We may therefore assume, in what follows, that $\chi \subset \zeta$.

If $|\zeta|=0$, i.e., $\zeta=\emptyset$, we must show that $\mathscr{S}_{1}(\emptyset) f=\mathscr{S}_{2}(\emptyset) f$; this follows from the observation above that $\mathscr{S}(\emptyset)=1$. We now consider a general $\zeta$. If $\chi \varsubsetneqq \zeta,(3.18)$ follows from the induction assumption. For from (3.16) (in the notation of Definition 3.5),

$$
f_{\lambda \zeta-x}=\Sigma f_{1}(\cdot, \mathscr{E})_{\lambda \zeta-x}=\Sigma f_{2}(\cdot, \mathscr{E})_{\lambda \zeta-x}
$$

for any $\lambda^{\zeta-\chi}$. From (3.15) and Definition 3.5,

$$
\mathscr{S}_{i}(\chi) f_{\lambda \zeta^{-\alpha}}(\lambda)=\mathscr{S}_{i}(\chi) f(\lambda), \quad(i=1,2) .
$$

But by the induction assumption, $\mathscr{S}_{1}(\chi) f_{\lambda^{\xi}-x}=\mathscr{S}_{2}(\chi) f_{\lambda^{\xi}-x}$, and this proves (3.18). Finally, if $\chi=\zeta,(3.18)$ follows from the $\chi \varsubsetneqq \zeta$ case and Corollary 3.12 , since (3.13) may be written in this case

$$
\mathscr{S}_{i}(\zeta) f=\mathscr{V} f-\sum_{\psi \subsetneq \zeta} \mathscr{S}_{i}(\psi) f .
$$

This completes the induction step, and proves Lemma 3.16.

Remark 3.19. For any $\chi \subset \Omega$, the operator $\mathscr{S}(\chi)$ satisfies

(a) $\mathscr{S}(\chi) f=0$ if, for some decomposition (3.3) of $f, f(\cdot, \mathscr{E})=0$ whenever $\overline{\mathscr{D}}=\chi$ for some $\mathscr{D} \subset \mathscr{E}$; then

(b) if $f=f_{1} f_{2}$, with $f_{i} \in \mathscr{B}$ depending only on $\lambda^{\chi_{i}}$, and $\chi_{1} \cap \chi_{2}=0$,

$$
\mathscr{S}(\chi) f=\left(\mathscr{S}\left(\chi \cap \chi_{1}\right) f_{1}\right)\left(\mathscr{S}\left(\chi \cap \chi_{2}\right) f_{2}\right) .
$$


Proof. (a) is immediate from Definition 3.14; (b) similarly (using Lemma 3.6) once we observe that each $f_{i}$ will have a decomposition (3.3) in which each $f_{i}(\cdot, \mathscr{E})$ depends only on $\lambda^{x_{i}}$.

The main result of this section now follows immediately from (3.13).

Theorem 3.20. For any analytic evaluator $\mathscr{V}$,

$$
\mathscr{V}=\sum_{\chi \subset \Omega} \mathscr{S}(\chi)
$$

We remark that (3.21) expresses the "regular part of $f$ ", $\mathscr{V} f$, in terms of $f$ itself together with subtractions of various singular parts (recall $\mathscr{S}(\emptyset)=1$ ). This is the natural form for renormalization theory. Of course, by transposing terms, (3.22) may be viewed as a decomposition of $f$ into various regular and singular parts.

\section{§ 4. Renormalization}

We now return to the study of the Feynman amplitude $\mathscr{T}_{G_{0}}$. Our complex variables are labelled by $\mathscr{L}=\mathscr{L}\left(G_{0}\right)$, and, as in $\S 2$, an $s$-family is a family of irreducible subgraphs of $G_{0}$. The lines of these subgraphs form an $s$-family in $\mathscr{L}$ in the sense of $\S 3$. We adapt the notation and speak of $\mathscr{S}(H)$, etc., rather than $\mathscr{S}\left(\mathscr{L}(H)\right.$ ), for $H$ any subgraph of $G_{0}$. We will show that (3.21), applied to $\mathscr{T}_{G_{0}}$, corresponds to an additive renormalization.

Definition 4.1. Suppose that $\left\{V_{1}^{\prime}, \ldots V_{m}^{\prime}\right\}$ is a generalized vertex of $G_{0}$, with $G=G\left(V_{1}^{\prime}, \ldots V_{m}^{\prime}\right)$. An IPI subgraph $H$ of $G_{0}$ is subordinate to $G(H \prec G)$ if $H$ also has vertices $V_{1}^{\prime}, \ldots V_{m}^{\prime}$ (we include the possibility $H=G$ ). Then

$$
\mathscr{X}\left(\lambda ; V_{1}^{\prime}, \ldots V_{m}^{\prime}\right)=\sum_{H<G} \mathscr{S}(H) \mathscr{T}_{G} .
$$

Lemma 4.2. $\mathscr{X}\left(\lambda ; V_{1}^{\prime}, \ldots V_{m}^{\prime}\right)$ is a vertex part for $\left\{V_{1}^{\prime}, \ldots V_{m}^{\prime}\right\}$.

Proof. If $G$ is not IPI, $\mathscr{X}=0$, since there exists no $H$ with $H \prec G$. Suppose then that $G$ is IPI, with irreducible components $G_{1}, \ldots G_{k}$. We wish to show that for any $H \prec G$ and any maximal $s$-family $\mathscr{E}$ of $G$, $\mathscr{S}(H) \mathscr{T}_{G}(\cdot, \mathscr{E})$ is, in momentum space, a factor $\delta\left(\Sigma p_{i}^{\prime}\right)$ times a polynomial in the $p_{i}^{\prime}$ of degree $\leqq \mu(G)$.

We use the explicit form of $\mathscr{T}_{G}(\lambda, \mathscr{E})$ : from [5],

$$
\mathscr{T}_{G}(\lambda, \mathscr{E})=\sum_{\sigma} \mathscr{T}_{G}(\lambda, \mathscr{E}, \sigma),
$$

the sum running over all maps $\sigma: \mathscr{E} \rightarrow \mathscr{L}(G)$ such that, for any $H \in \mathscr{E}$ and $H^{\prime} \in \mathscr{E}(H), \sigma(H) \in \mathscr{L}(H)$ and $\sigma(H) \notin \mathscr{L}\left(H^{\prime}\right) . \mathscr{T}_{G}(\lambda, \mathscr{E}, \sigma)$ is itself a 
sum of terms of the form

$$
\begin{aligned}
\lim _{\varepsilon \rightarrow 0^{+}} \delta( & \left.\sum_{i=1}^{m} p_{i}^{\prime}\right) \int_{D} \ldots \int\left[\prod_{H \in \mathscr{E}} t^{\nu_{H}-1} d t_{H}\right]\left[\prod_{\ell \in \sigma(\mathscr{E})} \beta^{\lambda_{\ell}-1} d \beta_{\ell}\right] \\
& \times F\left(\beta, t, p^{\prime}, m\right) \exp i\left[\sum_{i, j=1}^{m} p_{i} A_{i j}(\beta, t) p_{j}-\sum_{\ell \in \mathscr{L}(G)}\left(m_{\ell}^{2}-i \varepsilon\right) B_{\ell}(\beta, t)\right] .
\end{aligned}
$$

The integration region $D$ is $\left\{0 \leqq t_{G_{i}} \leqq \infty, 0 \leqq t_{H} \leqq 1\right.$ for $H \in \mathscr{E}\left(G_{i}\right)$, $\left.0 \leqq \beta_{\ell} \leqq 1\right\} . F, A_{i j}$, and $B_{\ell}$ are continuous functions in $D$, with $F$ a polynomial in the $p_{i}^{\prime}$ whose degree we denote $r$. For $H \in \mathscr{E}$,

$$
v_{H}=\Lambda(H)-k_{H},
$$

where $k_{H}$ is an integer. If $H \prec G$ has irreducible components $H_{1}, \ldots H_{k}$, then

in addition,

$$
2 \sum_{i=1}^{k} k_{H_{i}} \leqq \mu(G)-r
$$

$$
\left.A_{i j}(\beta, t)\right|_{t_{H_{l}}=0, i=1, \ldots k}=0 .
$$

This last result is not stated explicitly in [5] but follows easily from, e.g., (3.14) of that work.

The integral (4.4) actually converges only for $v_{H}>0$, but may be treated at the point $\lambda_{0}$ by considering each factor $t_{H}^{\nu_{H}-1}$ as a distribution and using the standard analytic continuation of that distribution [9]. (This method of analytic continuation of (4.4) is that of Westwater ([6]), not that of [5].) $t_{H}^{\nu_{H}-1}$ is, from (4.5), analytic at $\lambda_{0}$ unless $k_{H} \geqq 0$, in which case it has near $\lambda_{0}$ the form

$$
t_{H}^{v_{H}-1}=\frac{\delta^{\left(k_{H}\right)}\left(t_{H}\right)}{k_{H} ! \Lambda(H)}+(\text { regular part }) .
$$

Now take $H_{0} \prec G$; we wish to apply $\mathscr{S}\left(H_{0}\right)$ to (4.4). By Remark 3.19 (a), this gives 0 unless $\mathscr{E}$ contains all irreducible components $\left\{H_{1}, \ldots H_{k}\right\}$ of $H_{0}$. Suppose that it does. If we rewrite each factor $t_{H_{i}}^{v_{H_{i}}-1}$ in (4.4) according to (4.8), Remark 3.19 (a) implies that every term in the resulting sum is annihilated by $\mathscr{S}\left(H_{0}\right)$, except the one involving $\Pi_{i=1}^{k} \delta^{\left(k_{H_{i}}\right)}\left(t_{H_{i}}\right)$. But, from (4.6) and (4.7), this term is already a polynomial in the $p_{i}^{\prime}$ of degree $\leqq \mu(G)$, multiplied by $\delta\left(\Sigma p_{i}^{\prime}\right)$. Since $\mathscr{S}\left(H_{0}\right)$ will preserve this form, the lemma is proved.

Now let $H$ be a subgraph of $G_{0}$ which is the union of its irreducible components (this is not a trivial restriction on $H$; it is equivalent to requiring that each connected component of $H$ be IPI). Let $H_{1}, \ldots H_{p}$ be the connected components of $H$, and let $H_{i}$ have vertices $V_{i 1}^{\prime}, \ldots V_{i m(i)}^{\prime}$. 
Then we say that $H$ is subordinate to the partition $P(H \prec P)$ of $\left\{V_{1}, \ldots V_{m}\right\}$ into subsets $\left\{V_{i 1}^{\prime}, \ldots V_{i m(i)}^{\prime}\right\}, i=1, \ldots p$, and $\left\{V_{j}\right\}, V_{j} \notin\left\{V_{i 1}^{\prime}, \ldots V_{p m(p)}^{\prime}\right\}$.

Lemma 4.9. With the above notation, and with $\mathscr{X}$ given by Def. 4.1,

$$
\mathscr{T}_{P, \mathscr{X}}(\lambda)=\sum_{H<P} \mathscr{S}(H) \mathscr{T}_{\mathrm{G}_{0}}(\lambda) .
$$

Proof. Write $G_{i}=G\left(V_{i 1}^{\prime}, \ldots V_{i m(i)}^{\prime}\right)$; clearly $H_{i} \prec G_{i}$ for all $i$. According to Definitions 3.5 and $3.14, \mathscr{S}(H) \mathscr{T}_{G_{0}}$ is calculated by holding all $\lambda_{\ell}$ constant for $\ell \in \mathscr{L}^{\prime}=\mathscr{L}-\bigcup_{1}^{P} \mathscr{L}\left(G_{i}\right)$. We may choose these constants to have large real part, in which case $\mathscr{T}_{G_{0}}$ is a product

$$
\mathscr{T}_{G_{0}}=\prod_{\mathscr{L}^{\prime}} \Delta_{\ell} \prod_{i=1}^{P} \mathscr{T}_{G_{i}}
$$

Then from Remark 3.19 (b),

$$
\mathscr{S}(H) \mathscr{T}_{G_{0}}=\prod_{\mathscr{L}^{\prime}} \Delta_{\ell} \prod_{i=1}^{p} \mathscr{S}\left(H_{i}\right) \mathscr{T}_{G_{i}} .
$$

Summing over $H \prec P$ gives (4.10).

We note that if some connected component of $H$ is not IPI, then $\mathscr{S}(H) \mathscr{T}_{G_{0}}=0$, by Remark 3.19 (a).

We may now state the main theorem.

Theorem 4.11. Any analytic renormalization is an additive renormalization.

Proof. The analytically renormalized amplitude is $\mathscr{W}_{G_{0}}$, for some generalized evaluator $\mathscr{W}$. By Theorem A.5 (or by Remark 3.4 (b), if $\mathscr{W}$ is the standard generalized evaluator) there exists an analytic evaluator $\mathscr{V}$ with $\mathscr{W} f=(\mathscr{V} f)\left(\lambda_{0}\right)$. Construct vertex parts $\mathscr{X}$ from $\mathscr{V}$ using Definition 4.1. Then,

$$
\begin{aligned}
\mathscr{W} \mathscr{T}_{G_{0}} & =\left(\mathscr{V} \mathscr{T}_{G_{0}}\right)\left(\lambda_{0}\right) \\
& =\left[\sum_{H} \mathscr{S}(H) \mathscr{T}_{G_{0}}\right]\left(\lambda_{0}\right) \\
& =\lim _{\lambda \rightarrow \lambda_{0}} \sum_{P} \mathscr{T}_{P, \mathscr{C}}(\lambda),
\end{aligned}
$$

where we have use Theorem 3.20 and Lemma 4.9. This completes the proof.

\section{Appendix}

In this appendix we state and prove the converse of the relation between generalized and analytic evaluators noted in Remark 3.4 (a). The proof is quite similar to standard proofs of the equivalence of 
analytic renormalization with other schemes (see, e.g., $[5,6,8])$. We use the notation of $\S 3$.

Lemma A.1. Take $\chi \subset \Omega$ and $f \in \mathscr{B}$. Suppose that the variety $\{\Lambda(\psi)=0\}$ is a singularity of $\mathscr{S}(\chi) f$. Then necessarily either (i) $\psi \subset \chi$, or (ii) $\psi \cap \chi=\emptyset$.

Proof. Let $f(\cdot, \mathscr{E})$ be a term in the decomposition (3.3) of $f$, with $\overline{\mathscr{D}}=\chi$ for some discrete $\mathscr{D} \subset \mathscr{E}$. Any $\zeta \in \mathscr{E}$ satisfies either (a) $\zeta \subset \chi$, or (b) $\zeta \cap \chi=\overline{\mathscr{F}}$, for some $\mathscr{F} \subset \mathscr{D}$, and $\zeta \neq \overline{\mathscr{F}}$. Let

$$
f(\lambda, \mathscr{E})=g(\lambda, \mathscr{E}) / \Lambda(\mathscr{E})
$$

For each $\zeta \in \mathscr{E}$ of type (b) above, write

$$
\frac{1}{\Lambda(\zeta)}=\frac{1}{\Lambda(\zeta-\overline{\mathscr{F}})}-\sum_{\psi \in \mathscr{F}} \frac{\Lambda(\psi)}{\Lambda(\zeta) \Lambda(\zeta-\overline{\mathscr{F}})},
$$

and insert (A.3) into (A.2). When $\mathscr{S}(\chi)$ is applied to the result, Remark 3.19 (a) shows that all terms except the first are annihilated. Thus (using Remark 3.19 (b))

$$
\mathscr{S}(\chi) f=\sum_{\mathscr{E}}\left[\prod \frac{1}{\Lambda(\zeta-\overline{\mathscr{F}})}\right] \mathscr{S}(\chi)\left[\frac{g(\cdot, \mathscr{E})}{\Lambda(\mathscr{D} \cup \mathscr{E}(\mathscr{D}))}\right] .
$$

Definition 3.14 immediately shows that, aside from the $\Lambda(\zeta-\overline{\mathscr{F}})$ singularities explicitly displayed, (A.4) can contain only singularities $\{\Lambda(\psi)=0\}, \psi \subset \chi$. This completes the proof.

We now give the main theorem.

Theorem A.5. Let $\mathscr{W}$ be a generalized evaluator. Then there exists an analytic evaluator $\mathscr{V}$ such that, for $f \in \mathscr{B}, \mathscr{W} f=\mathscr{V} f\left(\lambda_{0}\right)$.

Proof. We use the analytic evaluator $\mathscr{V}_{0}$ constructed explicitly in Remark 3.4 (b), and denote singular parts defined with it by $\mathscr{S}_{0}$. We also note that we may define operators $\mathscr{W}_{\chi}$, for $\chi \subset \Omega$, exactly as we defined operators $\mathscr{V}_{\chi}$ in Definition 3.5. For example, $\mathscr{W}_{\chi} f$ is independent of $\lambda^{x}$, for any $f \in \mathscr{B}$.

We now define $\mathscr{V}$ by

$\mathscr{V}=\sum_{k=0}^{M} \sum_{\chi_{1}, \cdots \chi_{k}}(-1)^{k} \mathscr{V}_{0, \Omega-u \chi_{i}} \mathscr{W}_{\chi_{k}} \mathscr{S}_{0}\left(\chi_{k}\right) \mathscr{W}_{\chi_{k-1}} \ldots \mathscr{W}_{\chi_{1}} \mathscr{S}_{0}\left(\chi_{1}\right)$

Here the sum is over all (ordered) $k$-tuples $\chi_{1}, \ldots \chi_{k}$ of non-empty, pairwise disjoint subsets of $\Omega$. The $k=0$ term of (A.6) is understood to be simply $\mathscr{V}_{0}$. We claim that $\mathscr{V}$ is an analytic evaluator.

First note that, for $f \in \mathscr{B}, \mathscr{V} f$ is analytic at $\lambda_{0}$. This is because (V 6) (applied to $\left.\mathscr{V}_{0}\right)$ shows that $\mathscr{W}_{\chi_{k}} \mathscr{S}_{0}\left(\chi_{k}\right) \ldots \mathscr{S}_{0}\left(\chi_{1}\right) f$ is independent of $\lambda \cup \chi_{2}$, and the factor $\mathscr{V}_{0, \Omega-\cup \chi_{i}}$ then yields a regular function. Also, $\mathscr{V}$ clearly satisfies (V 1)-(V 4) and (V 6), so there remains to verify only 
(V 5). Suppose then that $f^{\prime}, f^{\prime \prime} \in \mathscr{B}$ depend only on $\lambda^{\psi^{\prime}}, \lambda^{\psi^{\prime \prime}}$ respectively, with $\psi^{\prime} \cap \psi^{\prime \prime}=\emptyset$. We apply $\mathscr{V}$ to $f^{\prime} f^{\prime \prime}$. By Remark 3.19 (b) and Lemma 3.6 (which applies to $\mathscr{W}$ just as to $\mathscr{V}_{0}$ ),

$$
\begin{aligned}
\mathscr{V}\left(f^{\prime} f^{\prime \prime}\right)= & \sum_{k=0}^{M} \sum_{\chi_{1}, \ldots \chi_{k}}(-)^{k}\left\{\mathscr{V}_{0, \psi^{\prime}-\cup \chi_{t}^{\prime}} \mathscr{W}_{\chi_{k}^{\prime}} \ldots \mathscr{S}_{0}\left(\chi_{1}^{\prime}\right) f^{\prime}\right\} \\
& \times\left\{\mathscr{V}_{0, \psi^{\prime \prime}-\cup \chi_{i}^{\prime \prime}} \mathscr{W}_{\chi_{k}^{\prime \prime}} \ldots f^{\prime \prime}\right\}
\end{aligned}
$$

where $\chi_{i}^{\prime}=\chi_{i} \cap \psi^{\prime}, \chi_{i}^{\prime \prime}=\chi_{i} \cap \psi^{\prime \prime}$. Now let $\left(\zeta_{1}^{\prime}, \ldots \zeta_{p}^{\prime}\right)$ and $\left(\zeta_{1}^{\prime \prime}, \ldots \zeta_{q}^{\prime \prime}\right)$ be the sequences $\left(\chi_{1}^{\prime}, \ldots \chi_{k}^{\prime}\right)$ and $\left(\chi_{1}^{\prime \prime}, \ldots \chi_{k}^{\prime \prime}\right)$ with all occurrences of the empty set deleted. Since $\mathscr{S}(\emptyset)=\mathscr{W}_{\emptyset}=1$,

$$
\mathscr{V}_{0, \psi^{\prime}-\cup \chi_{i}^{\prime}} \mathscr{W}_{\chi_{k}^{\prime}} \ldots \mathscr{S}_{0}\left(\chi_{1}^{\prime}\right) f^{\prime}=\mathscr{V}_{0, \psi^{\prime}-\cup \zeta^{\prime}} \mathscr{W}_{\zeta_{p}^{\prime}} \ldots \mathscr{S}_{0}\left(\zeta_{1}^{\prime}\right) f^{\prime},
$$

and similarly for $f^{\prime \prime}$ (again, the right hand side of (A.8) is $\mathscr{V}_{0, w^{\prime}} f^{\prime}$ when $p=0$, i.e., when $\chi_{i}^{\prime}=\emptyset$ for all i). We wish to rewrite (A.7) as a sum over $p, q, \zeta_{i}^{\prime}$, and $\zeta_{1}^{\prime \prime}$. A fixed $\left(\zeta_{1}^{\prime}, \ldots \zeta_{p}^{\prime}\right)$ and $\left(\zeta_{1}^{\prime \prime}, \ldots \zeta_{q}^{\prime \prime}\right)$ may come from many different $\left(\chi_{1}, \ldots \chi_{k}\right)$ terms in (A.7), but (because $\chi_{i} \neq \emptyset$ ) it is easy to see that $k$ must satisfy $p+q \geqq k \geqq \max (p, q)$, and that, for each such $k$, there are $\left(\begin{array}{l}k \\ p\end{array}\right)\left(\begin{array}{c}p \\ p+q-k\end{array}\right)$ such terms. Using

$$
\sum_{k=\max (p, q)}^{p+q}(-)^{k}\left(\begin{array}{l}
k \\
p
\end{array}\right)\left(\begin{array}{c}
p \\
p+q-k
\end{array}\right)=(-)^{p+q}
$$

the desired factorization follows immediately.

Eq. (A.9) itself may be derived by considering the coefficient of $x^{p} y^{q}$ in the equation

$$
\sum_{k, p, q=0}^{\infty}(-)^{k}\left(\begin{array}{l}
k \\
p
\end{array}\right)\left(\begin{array}{c}
p \\
p+q-k
\end{array}\right) x^{p} y^{q}=\frac{1}{(1+x)(1+y)},
$$

obtained by summing first over $q$, then $p$, then $k$.

There remains only to verify that $\mathscr{V} f\left(\lambda_{0}\right)=\mathscr{W} f$. We expand each $\mathscr{V}_{0}$ operator in (A.6) according to Theorem 3.20. After rearrangement, (A.6) becomes

$$
\mathscr{V}=\mathscr{S}_{0}(\emptyset)+\sum_{k=1}^{M} \sum_{\chi_{1}, \ldots \chi_{k}}(-)^{k+1}\left(1-\mathscr{W}_{\chi_{k}}\right) \mathscr{S}_{0}\left(\chi_{k}\right) \mathscr{W}_{\chi_{k-1}} \ldots \mathscr{W}_{\chi_{1}} \mathscr{S}_{0}\left(\chi_{1}\right)
$$

$\chi_{1}, \ldots \chi_{k}$ are as in (A.6). We apply $\mathscr{W}$ to (A.10). From Lemma 3.6 (applied to $\mathscr{W})$ and Lemma A.1,

$$
\begin{aligned}
& \mathscr{W}\left[\left(1-\mathscr{W}_{\chi_{k}}\right) \mathscr{S}_{0}\left(\chi_{k}\right) \mathscr{W}_{\chi_{k-1}} \ldots \mathscr{S}_{0}\left(\chi_{1}\right)\right] \\
& \quad=\mathscr{W}_{\chi_{k}} \mathscr{W}_{\Omega-v \chi_{i}}\left[\left(1-\mathscr{W}_{\chi_{k}}\right) \mathscr{S}_{0}\left(\chi_{k}\right) \mathscr{W}_{\chi_{k-1}} \ldots \mathscr{S}_{0}\left(\chi_{1}\right)\right],
\end{aligned}
$$


and this vanishes because $\mathscr{W}_{\chi_{k}}\left(1-\mathscr{W}_{\chi_{k}}\right)=0$. Thus $\mathscr{W} \mathscr{V}=\mathscr{W} \mathscr{S}_{0}(\emptyset)=\mathscr{W}$ But since, for $f \in \mathscr{B}, \mathscr{V} f$ is analytic at $\lambda_{0}, \mathscr{W} \mathscr{V} f=\mathscr{V} f\left(\lambda_{0}\right)$. This completes the proof.

\title{
Bibliography
}

1. Bogoliubov, N. N., Shirkov,D.V.: Introduction to the Theory of Quantized Fields. New York: Interscience Publ. Inc., 1959.

2. Hepp, K.: Commun. math. Phys. 2, 301 (1966).

3. - Thèorie de la renormalisation. Berlin-Heidelberg-New York: Springer 1969.

4. Speer, E. R.: J. Math. Phys. 9, 1404 (1968).

5. - Generalized Feynman Amplitudes. Princeton: Princeton University Press 1969.

6. Westwater, J.: Fortschr. Physik 17, 1 (1969).

7. Hepp, K.: Les Houches lectures, 1970.

8. - Commun. math. Phys. 14, 67 (1969).

9. Gel'fand,I.M., Shilov, G.E.: Generalized Functions, Vol. I. New York: Academic Press, Inc. 1964.

\author{
Eugene R. Speer \\ Department of Mathematics \\ Massachusetts Institute of Technology \\ Cambridge, Mass. 02139 USA
}

